“C 2019 IEEE. Personal use of this material is permitted. Permission from IEEE must be obtained for all other uses, in any current or future media, including reprinting/republishing this material for advertising or promotional purposes, creating new collective works, for resale or redistribution to servers or lists, or reuse of any copyrighted component of this work in other works." 


\section{A Comparison of Performance of GA, PSO and Differential Evolution Algorithms for Dynamic Phase Reconfiguration Technology of a Smart Grid}

\author{
Md Rabiul Islam, Haiyan (Helen) Lu. \\ School of Software \\ University of Technology Sydney \\ Broadway NSW 2007, Australia \\ MdRabiul.Islam-1@student.uts.edu.au \\ haiyan.lu@uts.edu.au
}

\author{
Md Jahangir Hossain \\ Department of Engineering \\ Macquarie University \\ NSW 2109, Australia \\ jahangir.hossain@mq.edu.au
}

\author{
$\mathrm{Li} \mathrm{Li}$ \\ Dept. of Electrical \& Data Engineering \\ University of Technology Sydney \\ Broadway NSW 2007, Australia \\ Li.Li@uts.edu.au
}

\begin{abstract}
Increasing penetration of Distributed Generations (Photovoltaic solar energy (PV), Wind energy, and Battery Energy Storage) and PEVs (Plug-in Electric Vehicles) into smart grid induce network imbalance which reduces power quality. The uncertainty of demand-generation requires balancing for mitigating network imbalance. Several researchers have used various optimization methods for mitigating unbalance. Moreover, a few researchers have done comparative studies of optimization methods for mitigating unbalance till now. This paper proposes a method to mitigate unbalance and reduce the total power loss by optimizing load distribution among phases. This paper compares the performance of Genetic Algorithm (GA), Particle Swarm Optimization (PSO) and Differential Evolution (DE) algorithms on the application of phase balancing. Finally, the efficacy of these algorithms are evaluated for the proposed unbalance mitigation technique, and it is found that the proposed technique using $D E$ algorithm can reduce a significant amount of unbalance at all the buses of the distribution grid with less computational effort.
\end{abstract}

Index Terms- Electric Vehicle, Unbalance Mitigation, GA, PSO, Differential Evolution, Smart Grid.

\section{INTRODUCTION}

The induction of smart monitoring system in smart grid provides valuable information's such as various losses, harmonic distortions, and level of voltage unbalance to distribution service operator for analyzing power quality, cost, generation-demand control and different types of fault [1]. The integration of PEVs and DGs into smart grid makes the smart grid more imbalance. The unequal distribution of residential and sudden EV loads integration among phases makes the certain phase more overloaded. Due to the quadratic nature of losses, the losses increase in the distribution grid. The network imbalance also increases grounding current which might be trip relays or breakers. Voltage imbalance reduces available capacity by increasing neutral current, higher voltage drop, minimize utilization of network asset which increases reinforcement cost [2-4].

Phase reconfiguration is a popular technology for balancing the grid. This technology changes the topology of the network using remote switches to satisfy the objective subject to constraints. Researchers consider total power loss [5], load balancing index [6], voltage deviation [7], current deviation [8], neutral current [9], and phase balancing index [10] as an objective function for phase balancing.

The author $[11,12]$ expressed the phase balancing problem as a non-linear integer problem, but the phase balancing problem cannot be expressed well as a linear problem. This study optimized the phase balancing problem using the simulated annealing method (SA). Though this study compares the performance of the SA method with the Greedy Algorithm and Quenching Algorithm, the SA algorithm requires higher computational time. Several researchers used heuristic algorithms to optimize phase balancing problems. The comparative study [13] for phase reconfiguration technique showed that Genetic Algorithm (GA) optimization method requires lower computational time than SA (Simulated Annealing), Backtracking algorithm [9], Exhaustive Search (ES), Greedy algorithm. The author [10] was used the GA optimization method to find optimum Phase reconfiguration for load balancing and power loss reduction. Another study [14] showed that GA is an efficient method to minimize the energy losses while the neutral current at the substation was an acceptable level. Another evolutionary algorithm PSO is used for solving phase reconfiguration technique. Evolutionary algorithms such as GA and PSO shows efficient ability to solve 
optimal distribution of loads, but the study [8] used the differential evolution (DE) algorithm. Moreover, none of the literature compares these optimization algorithms to identify the efficacy of the phase re-configuration technology. This paper investigates the efficacy of GA, PSO and DE algorithms for optimal phase reconfiguration technology.

Detail modeling of the test system, problem formulation and the proposed technique for mitigating unbalance is provided in Section II. An overview of GA, PSO and DE optimization methods for mitigating network imbalance is provided in Section III, and its performance is investigated in Section IV. Section V includes the summary and future direction of this research work.

\section{PROPOSED UNBALANCE MITIGATION TECHNIQUE}

\section{A. Test system}

In this study, the power quality performance of low voltage (LV) residential distribution feeder with 13 bus is investigated. The LV distribution grid is connected with distributed generation sources (PV, Wind, and Battery Energy Storage) and single phase PEVs which are connected to the main grid through a transformer rated at $10 \mathrm{MVA}, 11 \mathrm{kV} / 0.4 \mathrm{kV}$ as shown in Fig. 1. The increasing penetration of DG and EV into LV distribution grid shows a higher degree of unbalance which violates voltage constraints, reduces network hosting capacity and energy losses [15]. The residential and EV loads are connected through Load Selector Switch to the phases. The unequal distribution of loads among phases decreases the network quality of the LV distribution grid.

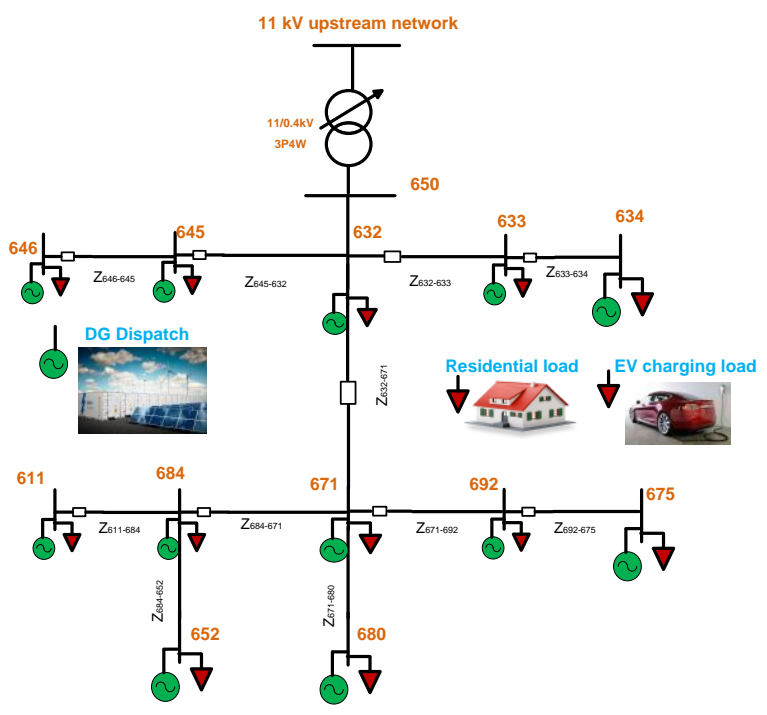

Fig.1. IEEE 13 Bus distribution system.

\section{B. Problem formulation}

This sub-section deals with the formulation of the problem. The objective function and constraints are formulated. The objective function includes voltage unbalance factor and the total power loss of the network as elaborated below:

$$
\begin{aligned}
& \gamma_{1}=V U F=\frac{\left|V_{-}\right|}{\left|V_{+}\right|} \\
& \gamma_{2}=\delta P_{\text {loss }}=\frac{P_{\text {loss_opt }}}{P_{\text {loss_base }}}
\end{aligned}
$$

Where,

$\delta P_{\text {loss }}=$ The power loss index.

$P_{\text {loss_base }}=$ The total power loss of the existing network.

$P_{\text {loss_opt }}=$ The total power loss after the execution of the proposed network.

$$
f_{\text {EUMT }}=p \gamma_{1}+q \gamma_{2}
$$

Where $p, q=$ weighting factor which will be set by Distribution Service Operator (DSO).

The voltage unbalance factor (VUF) is defined as the ratio of negative sequence voltage to positive sequence voltage and shown in equation (1), and the total power loss index is shown in equation (2).

The $\mathrm{p}, \mathrm{q}$ is multi-objective weighting factor which will be decided by DSO considering planning/optimization criteria of the network. The operational constraints of DGs mean the amount of active and reactive power of each DG unit should be within their operational limit which are applicable for both synchronous and inverter based DGs. The active power constrained dispatched by each DG $\left(P_{D G}\right)$ is expressed as equation (4) and reactive power $\left(Q_{D G}\right)$ dispatched constrained shown in equation (5).

$$
\begin{aligned}
& P_{D G_{-} \min }{ }^{i}(t) \leq P_{D G}{ }^{i}(t) \leq P_{D G_{-} \max }{ }^{i}(t) \\
& Q_{D G_{-} \min }{ }^{i}(t) \leq Q_{D G^{i}}{ }^{i}(t) \leq Q_{D G_{-} \max }{ }^{i}(t)
\end{aligned}
$$

Where,

$$
\forall i \in N B u s \text { and } \forall t \in T
$$

The EVs are also connected to the LV distribution grid which can act as either in charging $\left(P_{E V_{-} c h}\right) /$ discharging $\left(P_{E V_{-} d c h}\right)$ mode at a specific hour (t). The constraints of EVs can be shown in below equation.

$$
\begin{aligned}
& P_{E V_{-} d c h \text { min }}{ }^{i}(t) \leq P_{E V_{-} d c h}{ }^{i}(t) \leq P_{E V_{-} d c h \max }{ }^{i}(t) \\
& P_{E V_{-} c h \min }^{i}(t) \leq P_{E V_{-} c h}{ }^{i}(t) \leq P_{E V_{-} c h \text { max }}{ }^{i}(t)
\end{aligned}
$$




$$
\begin{aligned}
& \operatorname{SOC}_{\min }^{m}(t) \leq \operatorname{SOC}^{m}(t) \leq S O C_{\max }^{m}(t) \\
& \operatorname{SOC}\left(m, t_{\text {initial }}\right)=\operatorname{SOC}(m), \forall t \in t_{\text {initial }} \\
& \operatorname{SOC}\left(m, t_{\text {final }}\right)=\operatorname{SOC}(m), \forall t \in t_{\text {final }}
\end{aligned}
$$

Where,

$\forall i \in N B u s, \quad \forall m \in N E V$ and $\forall t \in T$

The charging and discharging power of each EV at a specific time should be within the allowable limit of the vehicle as shown in equation (6) and (7). The initial and final value of the State of charge ( $S O C$ ) of the EV battery for charging or discharging a constrained which is as shown in equation (8$10)$.

The total power loss constraint can be defined that the total Power loss $\left(P_{\text {loss }}\right)$ should be lower than the re-configured network as shown in equation (11), and the bus voltage constraint also expressed for the re-configured network is expressed as in equation (12).

$$
\begin{aligned}
& P_{\text {loss_opt }} \leq P_{\text {loss_base }} \\
& V_{\text {avg_base }}{ }^{(i)} \leq V_{a v g}{ }^{(i)} \leq V_{s}^{(i)} \\
& V_{\text {avg_base }}{ }^{(i)}=\text { Bus voltage of the existing network. } \\
& \text { Vs = Slack bus voltage. }
\end{aligned}
$$

The objective function expressed in equation (3) subject to constraint (4-12) should be maintained during distribution network reconfiguration. The optimum phase reconfiguration for improving its dynamic performance is obtained using the proposed optimization technique which is described in the later section.

\section{Proposed technique}

This paper proposes a technique to mitigate the unbalance of a distribution grid. This technique will use re-phasing method for minimizing network imbalance as well as power quality of the network. The objective function as shown in equation (3) subject to constraint (4-12) will be minimized to mitigate unbalance. The optimal solution is obtained using evolutionary algorithms (GA, PSO, and DE). This paper also compares the performance of GA, PSO, and DE for re-phasing technique. The proposed tasks will be implemented and investigated using Digsilent Powerfactory. The proposed technique is described as follows-

Stage 1: Read data from the linked input file (CSV file format in the local drive of a computer); residential loads per phase,
DG dispatch, EV charging demand and EV discharging dispatch, line parameter, etc.

Stage 2: Set input parameters for evolutionary algorithms (GA, $\mathrm{PSO}$, and DE).

Stage 3: The unbalanced load flow is executed using the DPL capability of the Digsilent Powerfactory and calculates the bus voltage, the total power loss and the VUF.

Stage 4: The optimal load distribution among phases using evolutionary algorithms (GA, PSO, and DE) for optimal load distribution to minimize the objective function (3) subject to constraint (4-12).

Stage 5: The DPL script of stage $3 \& 4$ repeats until reached either the maximum iteration number or the fitness value is negative.

Stage 6: The reconfigured loads per phase per bus into the linked output file (CSV file format in the local drive of a computer) using the DPL script.

The proposed technique can be implemented using hierarchical decentralized technology [16-19] as shown in Fig.2. The decentralized framework collects required input data, calculate various performance indicators, and implement the proposed technique to determine the optimal network topologies.

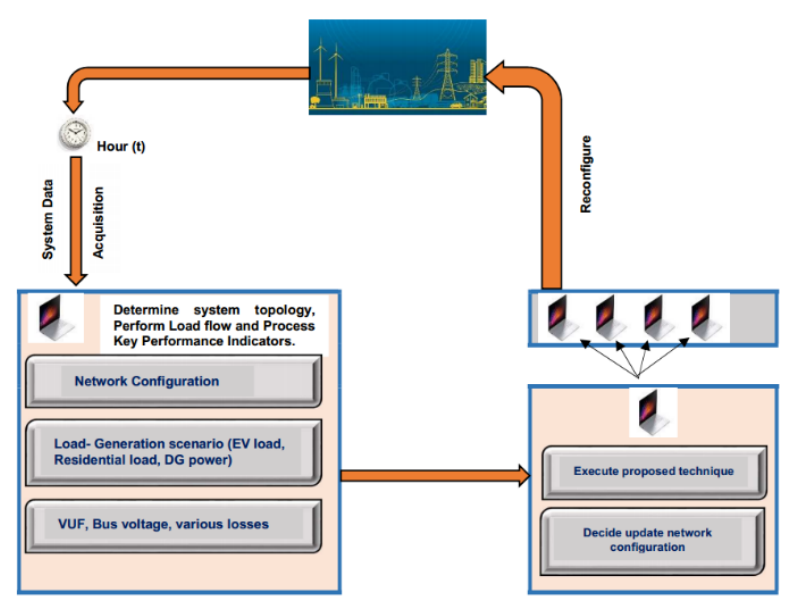

Fig.2. Framework of the Hierarchical Decentralized Distribution System.

\section{OPTIMIZATION ALGORITHMS}

\section{A. Genetic Algorithm (GA)}

GA is a biological evolutionary heuristic search algorithm for systematical optimization [20] . GA is useful where variables are discrete, and constraints are explicit. GA will generate randomly a population of strings conforming to the status of the system condition. Parameters of GA must be set initially such as generation size, population size, crossover probability, and mutation probability. From those seed strings, GA will 
perform its search. A chromosome string is used in this paper to represent loads distribution among phases whereas gene represents loads in phase $\mathrm{A}$, phase $\mathrm{B}$ and phase $\mathrm{C}$. To confine the searching space, the gene is encoded as $0,1,2$ to represent loads in phase A, phase B and phase C. Each string represents a system status and will lead to minimizing the fitness function subject to the constraints. This string will be discarded when load flow is divergent, or the fitness function value turns negative. The Genetic Algorithm GA can be summarized as shown in algorithm-I.

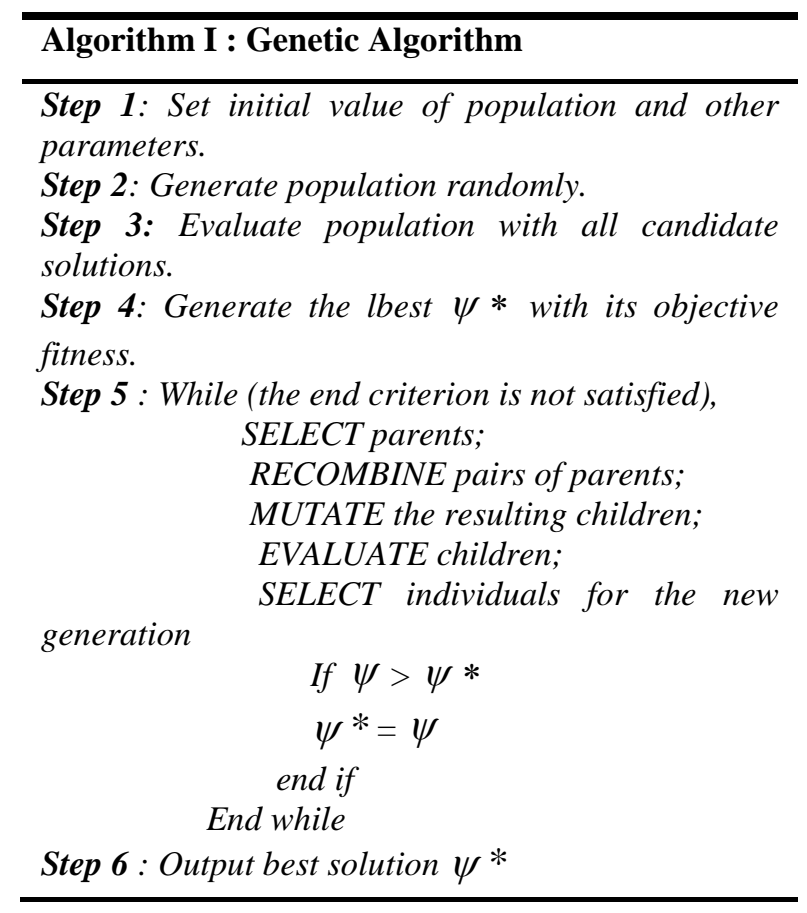

\section{B. Particle Swarm Optimization (PSO)}

In this study, the particle is a vector which comprises the elements that need to be optimized (say, loads per bus). Swarm is a matrix of particles. Every element is called a swarm member in this study. The algorithm starts with a random swarm of particles and a random speed matrix which has the same dimension as the swarm. The speed matrix takes random values between 0 and 1 .

For a certain number of generations, every particle is evaluated according to the fitness function by satisfying the constraints. The objective of the proposed algorithm is to find the optimum loads per phase to obtain the lower VUF value. After all the particles from one generation are evaluated, it is created the PBest matrix and GBest vector. PBest is the matrix with the best performance of every particle until the generation $\mathrm{j}$ (current iteration). GBest is the vector with the best particle ever discovered until the generation $\mathrm{j}$. The best particle is reintroduced into the swarm if it doesn't exist anymore. The algorithm will compute the new position of the particle and repeat the process until reaching the maximum number of generation. The main steps of the algorithm are summarized in algorithm-II.
Algorithm II : Particle Swarm Optimization Algorithm

Step I: Initialize the value of acceleration constants c1, $c 2$ and swarm size.

Step II: set the counter $\alpha=0$.

Step III: Generate random $x_{\gamma}{ }^{(\alpha)}$ and $v_{\gamma}{ }^{(\alpha)} \in[L, U]$

where $\gamma=1 \ldots S S$.

Step IV: Evaluate the fitness function $f\left(x_{\gamma}{ }^{(\alpha)}\right)$.

Step V: Set gbest ${ }^{(\alpha)}$.

[ gbest ${ }^{(\alpha)}$ is the best local solution in the swarm].

Step VI: Set pbest ${ }_{\gamma}{ }^{(\alpha)}$.

[ pbest $_{\gamma}{ }^{(\alpha)}$ is the best local solution in the swarm].

Step VII: Repeat.

StepVIII:

$v_{\gamma}{ }^{(\alpha+1)}=v_{\gamma}{ }^{(\alpha)}+C_{1} \times \operatorname{rand}_{1} \times\left(\right.$ pbest $\left._{\gamma}{ }^{(\alpha)}-x_{\gamma}{ }^{(\alpha)}\right)$

$+C_{2} \times \operatorname{rand}_{2} \times\left(\right.$ gbest $\left.^{\alpha}-x_{\gamma}^{(\alpha)}\right)$

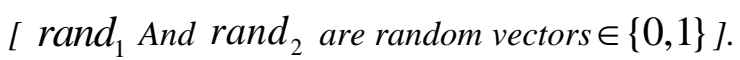

Step IX: $\quad x_{\gamma}{ }^{(\alpha+1)}=x_{\gamma}+v_{\gamma}{ }^{(\alpha+1)}, \gamma=1, \ldots \ldots \ldots . . . S S$.

[ Update particles positions].

Step X: Evaluate the fitness function $f\left(x_{\gamma}{ }^{(\alpha+1)}\right), \gamma=$ $1 \ldots . S S$.

Step XI: if $f\left(x_{\gamma}{ }^{(\alpha+1)}\right) \leq f\left(\right.$ pbest $\left._{\gamma}{ }^{(\alpha)}\right)$ then

$$
\text { pbest }_{\gamma}{ }^{(\alpha+1)}=x_{\gamma}{ }^{(\alpha+1)} \text {. }
$$

Else

$$
\operatorname{pbest}_{\gamma}^{(\alpha+1)}=\operatorname{pbest}_{\gamma}^{(\alpha)}
$$

End if

$$
\begin{gathered}
\text { if } x_{\gamma}{ }^{(\alpha+1)} \leq f\left(\text { gbest }^{(\alpha)}\right) \text { then } \\
\text { gbest }^{(\alpha+1)}=x_{\gamma}{ }^{(\alpha+1)} .
\end{gathered}
$$

Else

End if

$$
\operatorname{gbest}^{(\alpha+1)}=\operatorname{gbest}^{(\alpha)} .
$$

Step XII: increment iteration $\alpha=\alpha+1$ until satisfy the criteria.

Step XIII: Generate best particle. 


\section{Differential Evolution (DE)}

In 1997, Stron and Price proposed Differential evolution algorithm (DE). The solution of DE algorithm is presenting a $\mathrm{D}$ dimensional vector. DE generates random population with Population size $\mathrm{N}$ and $\mathrm{D}$ dimensional vector can be expressed as:

$x_{i}(t)=\left\{x_{i, 1}{ }^{(t)}, x_{i 2}(t), \ldots \ldots \ldots \ldots x_{i D}(t)\right\}, \quad[i=1,2 \ldots N]$

Where

$t=$ Number of generation

$D=$ Dimensional variable number

$N=$ population size

The generation of a trial vector is accomplished by the mutation and crossover operations. The mutant vector can be expressed as:

$v_{i}{ }^{(t)}=x_{\delta 1}{ }^{(t)}+F .\left(x_{\delta 2}-x_{\delta 3}\right)+F \cdot\left(x_{\delta 4}-x_{\delta 5}\right)$

Where,

The indexes $\delta d, d=1,2,3 \ldots \ldots \ldots . .5$ represent the random and mutually different integers generated within the range [ 1 , $N]$ and not equal to $i$.

$F=$ Mutation scaling factor within the range $[0,1]$.

The second step is to generate the trail vector by performing crossover between the mutant vector and the target vector which can be expressed as:

$$
\begin{aligned}
& u_{i j}=\begin{array}{l}
\{ \\
v_{i, j,} \text { if } \operatorname{random}(0,1) \leq C R \text { or } \\
j=j_{\text {random }}
\end{array} \\
& x_{i, j} \text {, Otherwise. } \\
& \}
\end{aligned}
$$

Where,

$C R=$ the crossover probability of creating parameters for a trail vector from the mutant vector and also known as control parameter within range $[0,1]$.

$j_{\text {random }}=$ random integer within range $[1, N]$.

The selection stage is to keep the vector with better fitness value between the trail and mutant vector. The definition of the selection operator as follows:

$$
x_{i}{ }^{(t+1)}=\begin{aligned}
& \left\{\begin{array}{l}
u_{i}{ }^{(t)} \quad \text { if } f\left(u_{i}{ }^{(t)}\right) \leq f\left(x_{i}{ }^{(t)}\right), \\
\left.x_{i}, \text { Otherwise. }\right\}
\end{array}\right.
\end{aligned}
$$

The Differential Evolution (DE) algorithm is summarize in Algorithm III.

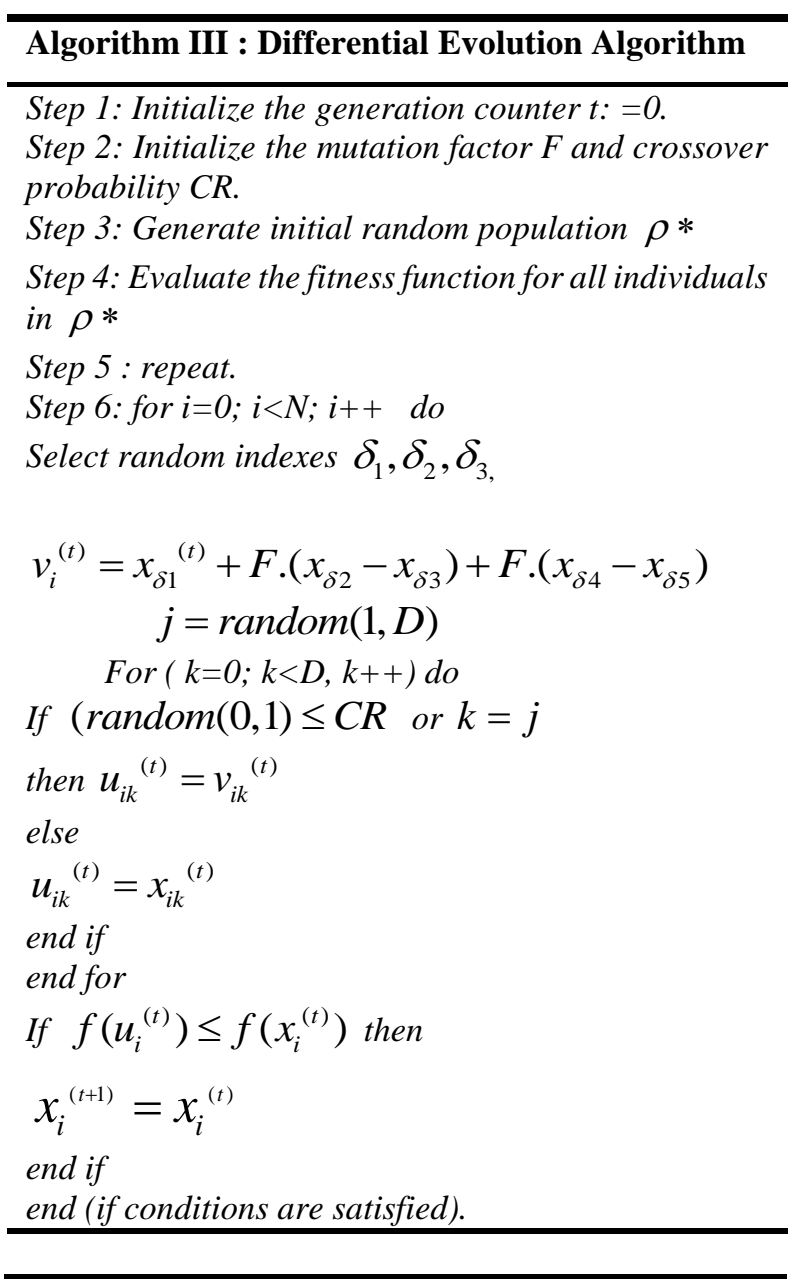

\section{RESULTS AND DISCUSSION}

In this section, the efficacy of the proposed technique and the performance of evolutionary algorithms for the optimal rephasing technique is discussed here. The LV distribution grid assumes higher penetration of EV charging loads for accessing impact on network performance. The optimal phase reconfiguration is solved through a proposed technique using evolutionary algorithms which is usually require less computational time than other classical methods [9] [10] [13].

The aim of this study is to find out suitable optimization algorithms among GA, PSO, and DE. To find out the suitable optimization parameter for each evolutionary algorithm, The GA, PSO and DE algorithm runs 100 iterations with 50 population. The parameter setting for evolutionary algorithms is crucial, and Fig, $2 \& 3$ presents fitness values based on crossover and mutation probability. Fig. 3 shows the minimum fitness values is observed during crossover=0.7-0.9 and mutation=0.1-0.3 for GA while solving the proposed rephasing technique. The best fitness is obtained while the value of crossover $=0.8$, and mutation $=0.2$. 
Fig. 4 shows that the minimum fitness values observed during $C R=0.6$ to 0.9 and $F=0.1$ to 0.3 during solving the proposed technique using the DE algorithm. The best fitness is obtained while the value of $C R=0.9$, and $F=0.2$. In PSO, the c1 (0.3), c2 (0.35), and inertia weight $(0.75)$ are selected while the best fitness is obtained.

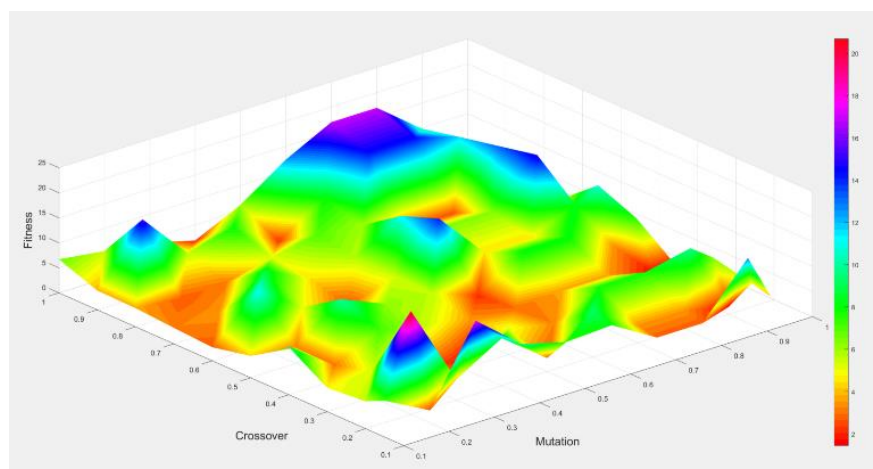

Fig.3. GA fitness, varying the crossover and mutation values for the proposed optimal re-phasing technique. The best fitness is obtained while crossover $=0.8$, mutation $=0.2$.

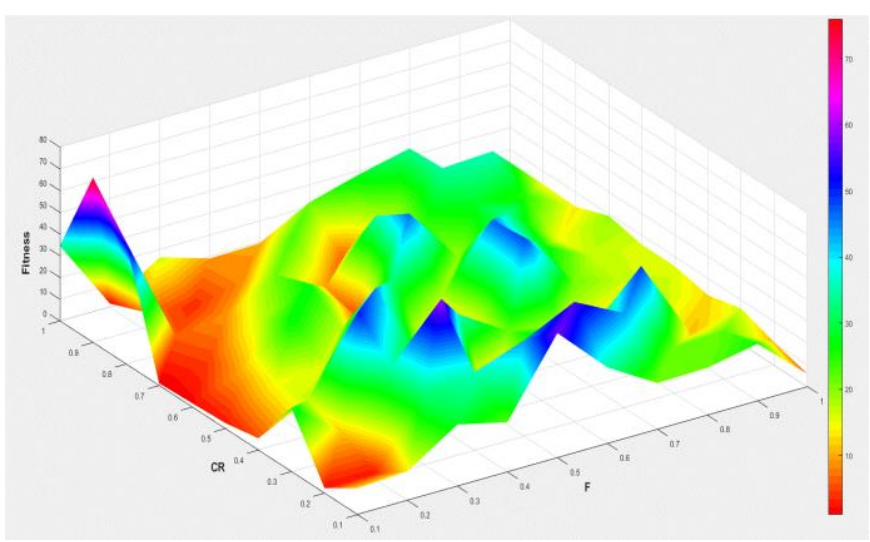

Fig.4. DE fitness, varying the $\mathrm{CR}$ and $\mathrm{F}$ values for the proposed optimal rephasing technique. The best fitness is obtained while $\mathrm{CR}=0.9, \mathrm{~F}=0.2$.

Total of thirty experiments conducted considering 10,000 evaluations to obtain the best solution using obtained optimized parameters of GA, PSO, and DE. For each algorithm, 50 population considered and analyze the best solution (optimum value) to compare the efficacy of evolutionary algorithms (GA, PSO, and DE) for the proposed optimal re-phasing technique. The analysis considers not only the relation of the number of evolutions and average fitness (convergence) but statistical indicators. The number of evaluation $(\mathfrak{J})$ is defined as the multiplication factor of number of population $(\rho)$ and number of iteration/generation $(\xi)$.

$$
\mathfrak{J}=\rho \times \xi
$$

The performance of evolutionary algorithms for the proposed technique could be determined to find out the optimal values of equation (3) subject to constraints (4-12). The Fig.5 shows a comparison of three algorithms convergence during performing the proposed technique.

The Fig.5 shows the DE algorithm converge to the minimum allowable value (0) at 75 iterations whereas PSO requires 150 iterations and GA is too far to achieve convergence. So, the DE shows faster convergence speed than GA and PSO while performing the proposed re-phasing technique. Apart from graphical analysis, the standard deviation values shown in Table I establish a better comparison analysis. The statistical analysis results are presented through a box plot using GA, PSO, and DE whose variables were calculated from the obtained fitness data at each iteration with 50 individuals. The statistics data are listed in Table I.

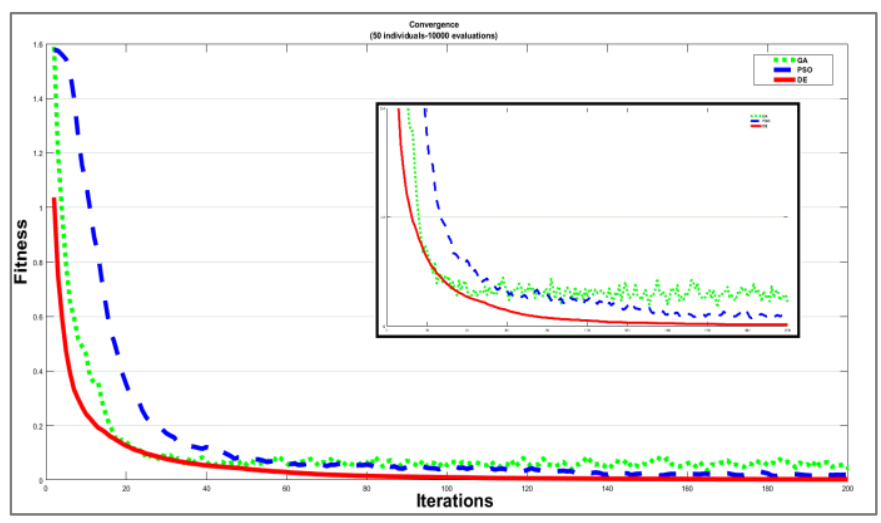

Fig.5. Convergence of GA, PSO and DE algorithms while performing proposed re-phasing technique.

TABLE I. STAISTICS OF THE OPTIMIZATION METHODS

\begin{tabular}{|l|l|l|l|}
\hline \multirow{2}{*}{$\begin{array}{l}\text { Statistical } \\
\text { indicators }\end{array}$} & \multicolumn{3}{|c|}{ Optimization Methods } \\
\cline { 2 - 4 } & GA & PSO & DE \\
\hline Minimum & 0.000606 & 0.001862 & 0.000672 \\
\hline $1^{\text {st }}$ quartile & 0.0010995 & 0.0021005 & 0.0010145 \\
\hline Median & 0.0069497 & 0.0040356 & 0.00179125 \\
\hline $3^{\text {rd }}$ quartile & 0.004759 & 0.006295 & 0.003244 \\
\hline Maximum & 0.041641 & 0.008588 & 0.003768 \\
\hline
\end{tabular}

The Fig.6 is constructed with data from Table I and shows the comparative performances of three algorithms for the proposed re-phasing technique. Fig. 6 shows that all statistical indicators have reduced to a point meaning higher homogeneity around the median. From Fig.6, it is observed that the DE indicate a greater homogeneity than GA and PSO. The DE also shows higher computational speed while solving the proposed technique. The performance of the proposed technique also investigated using these algorithms, and worst bus performance was listed in Table II. 
The efficacy of the DE algorithm shows better than GA and PSO. The performance of the proposed technique using the DE algorithm is compared with existing network performance as shown in Fig. 6, 7 \& 8 .

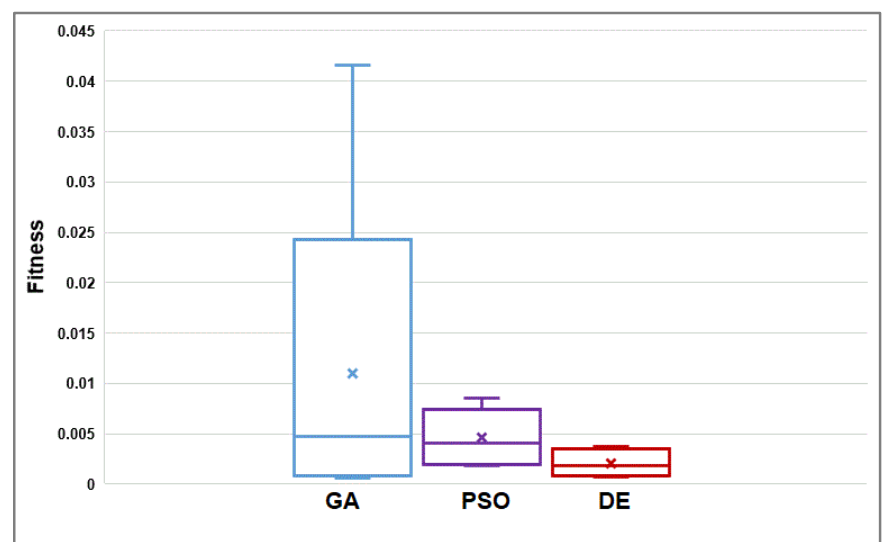

Fig.6. Statistical performance of GA, PSO and DE algorithms during performing proposed re-phasing technique.

TABLE II. GRID PERFORMANCES OF THE OPTIMIZATION METHODS

\begin{tabular}{|c|c|l|l|l|}
\hline \multirow{2}{*}{$\begin{array}{l}\text { Key } \\
\text { Performance } \\
\text { Indicators }\end{array}$} & \multirow{2}{*}{$\begin{array}{c}\text { Bus } \\
\text { Name }\end{array}$} & \multicolumn{3}{|c|}{ Methodologies } \\
\cline { 3 - 5 } $\begin{array}{c}\text { Bus Voltage } \\
\text { (p.u) }\end{array}$ & Bus 652 & 0.9120 & 0.9062 & 0.9410 \\
\cline { 2 - 5 } & Bus 680 & 0.9255 & 0.9181 & 0.9565 \\
\hline VUF (\%) & Bus 652 & 6.0592 & 6.6935 & 3.2198 \\
\cline { 2 - 5 } & Bus 680 & 3.9206 & 4.5305 & 0.4783 \\
\hline $\begin{array}{c}\text { Voltage Drop } \\
\text { along Feeder } \\
(\%)\end{array}$ & Bus 652 & 8.7976 & 9.3763 & 5.8969 \\
\cline { 3 - 5 } & Bus 680 & 7.4474 & 8.1819 & 4.3448 \\
\hline
\end{tabular}

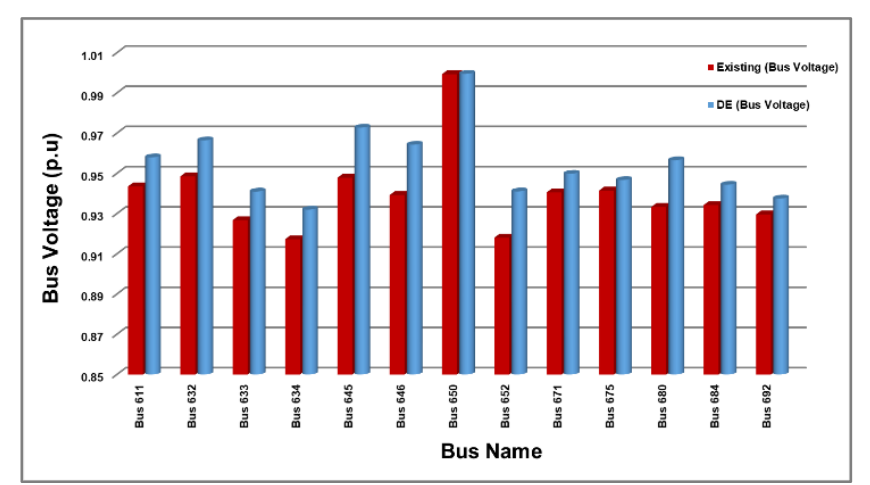

Fig.7. Improvement of bus voltage (p.u) using the proposed technique with DE.

The proposed technique shows that VUF is reduced to almost $3 \%$ which is also standard for the network operators. The total real power loss of the network also reduces from $166.52 \mathrm{~kW}$ to $126.77 \mathrm{~kW}$ and reactive power loss from $813.02 \mathrm{kvar}$ to 705.2 kvar. The reduction of network imbalance and minimizing of power loss not only improve power quality but also reduces voltage drop along feeder and the bus voltage. Both the technical and computational performances of these three algorithms are discussed, and it is found that the DE algorithms show better performance for solving optimal re-phasing technique.

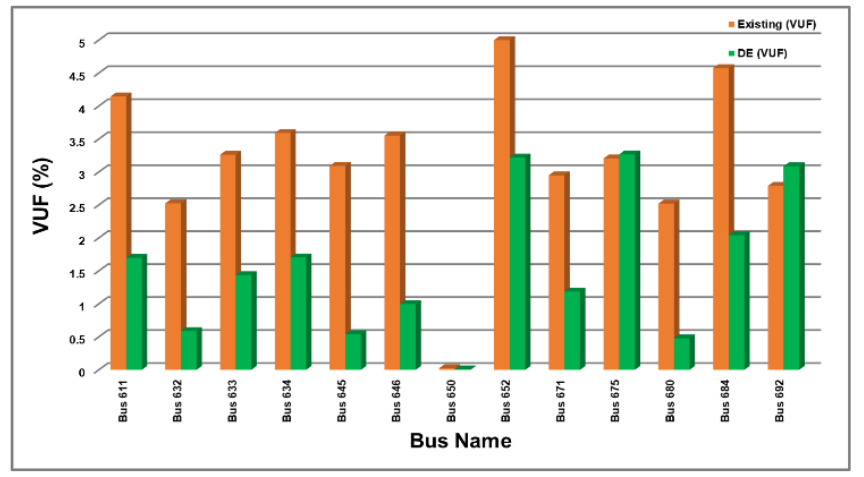

Fig.8. Improvement of VUF (\%) using the proposed technique with DE

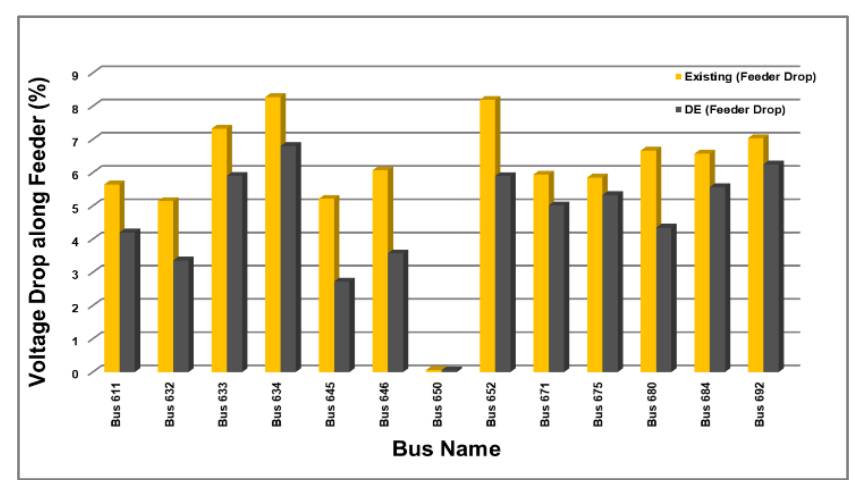

Fig.9. Improvement of voltage drop along feeder (\%) using the proposed technique with DE.

\section{CONCLUSION}

From the above discussion, it is observed that the proposed technique improves the power quality of the distribution grid by mitigating unbalance, reducing total power loss and voltage drop, and increase bus voltage. The comparison of the three optimizations (GA, PSO, and DE) method is applied to solve the proposed optimal re-phasing technique by following constraints. The DE algorithm shows greater homogeneity around the mean compared to GA and PSO. PSO also shows more effective performance than GA. However, DE is more robust and consistent than GA and PSO. The DE algorithm not only improves power quality but also require less computational speed. Therefore, these results suggest that the efficient performance is obtained using the DE algorithm and more detailed investigation will be done in the future for solving similar kind of problems. 


\section{REFERENCES}

[1] S. S. S. R. Depuru, L. Wang, and V. Devabhaktuni, "Smart meters for power grid: Challenges, issues, advantages and status," Renewable and Sustainable Energy Reviews, vol. 15, no. 6, pp. 2736-2742, 2011/08/01/2011.

[2] K. Ma, R. Li, and F. Li, "Quantification of Additional Asset Reinforcement Cost From 3-Phase Imbalance," IEEE Transactions on Power Systems, vol. 31, no. 4, pp. 2885-2891, 2016.

[3] M. K. Gray and W. G. Morsi, "Economic assessment of phase reconfiguration to mitigate the unbalance due to plug-in electric vehicles charging," Electric Power Systems Research, vol. 140, no. Supplement C, pp. 329-336, 2016/11/01/ 2016.

[4] M. Sadeghi and M. Kalantar, "The analysis of the effects of clean technologies from economic point of view," Journal of Cleaner Production, vol. 102, no. Supplement C, pp. 394-407, 2015/09/01/ 2015.

[5] N. S. Galat and P. M. Sonawane, "Distribution system feeder reconfiguration by robust optimization method, objectives and solution methods," in 2017 International Conference on Intelligent Computing and Control Systems (ICICCS), 2017, pp. 248-251.

[6] I. I. Atteya, H. Ashour, N. Fahmi, and D. Strickland, "Radial distribution network reconfiguration for power losses reduction using a modified particle swarm optimisation," CIRED - Open Access Proceedings Journal, vol. 2017, no. 1, pp. 2505-2508, 2017.

[7] A. Ukil and W. Siti, "Feeder load balancing using fuzzy logic and combinatorial optimization-based implementation," Electric Power Systems Research, vol. 78, no. 11, pp. 1922-1932, 2008/11/01/ 2008 .

[8] M. Sathiskumar, A. Nirmal kumar, L. Lakshminarasimman, and S. Thiruvenkadam, "A self adaptive hybrid differential evolution algorithm for phase balancing of unbalanced distribution system," International Journal of Electrical Power \& Energy Systems, vol. 42, no. 1, pp. 91-97, 2012/11/01/ 2012.

[9] L. Chia-Hung, C. Chao-Shun, C. Hui-Jen, and H. Cheng-Yu, "Heuristic rule-based phase balancing of distribution systems by considering customer load patterns," IEEE Transactions on Power Systems, vol. 20, no. 2, pp. 709-716, 2005.

[10] C. Tsai-Hsiang and C. Jeng-Tyan, "Optimal phase arrangement of distribution transformers connected to a primary feeder for system unbalance improvement and loss reduction using a genetic algorithm," IEEE Transactions on Power Systems, vol. 15, no. 3, pp. 994-1000, 2000.

[11] J. Zhu, G. Bilbro, and C. Mo-Yuen, "Phase balancing using simulated annealing," IEEE Transactions on Power Systems, vol. 14, no. 4, pp. 1508-1513, 1999.

[12] M. A. Kashem, V. Ganapathy, and G. B. Jasmon, "A geometric approach for three-phase load balancing in distribution networks," in PowerCon 2000. 2000 International Conference on Power System Technology. Proceedings (Cat. No.00EX409), 2000, vol. 1, pp. 293-298 vol.1.

[13] K. Wang, S. Skiena, and T. G. Robertazzi, "Phase balancing algorithms," Electric Power Systems Research, vol. 96, pp. 218224, 2013/03/01/ 2013

[14] I. P. Abril, "Genetic Algorithm for the Load Balance on Primary Distribution Circuits," IEEE Latin America Transactions, vol. 8, no. 5 , pp. 526-532, 2010.

[15] A. Rodriguez-Calvo, R. Cossent, and P. Frías, "Integration of PV and EVs in unbalanced residential LV networks and implications for the smart grid and advanced metering infrastructure deployment," International Journal of Electrical Power \& Energy Systems, vol. 91, no. Supplement C, pp. 121-134, 2017/10/01/ 2017.

[16] D. V. Nicolae, M. W. Siti, and A. A. Jimoh, "LV self balancing distribution network reconfiguration for minimum losses," in 2009 IEEE Bucharest PowerTech, 2009, pp. 1-6.

[17] F. Ding and K. A. Loparo, "Hierarchical Decentralized Network Reconfiguration for Smart Distribution Systems-Part I: Problem Formulation and Algorithm Development," IEEE Transactions on Power Systems, vol. 30, no. 2, pp. 734-743, 2015.
F. Ding and K. A. Loparo, "Hierarchical Decentralized Network Reconfiguration for Smart Distribution Systems-Part II: Applications to Test Systems," IEEE Transactions on Power Systems, vol. 30, no. 2, pp. 744-752, 2015.

[19] F. Ding and K. A. Loparo, "Feeder Reconfiguration for Unbalanced Distribution Systems With Distributed Generation: A Hierarchical Decentralized Approach," IEEE Transactions on Power Systems, vol. 31, no. 2, pp. 1633-1642, 2016.

[20] J. H. H. David E. Goldberg, "Genetic Algorithm in Search of Optimization and Machine learning," vol. 3, no. 2-3, pp. 95-99, October 1988. 